\title{
Assessment Using the ICF-core set for Children and Youth with Cerebral Palsy: A Single Case Study
}

\author{
YuNa Yun', GoEun Choi', Hyoung won Lim² \\ 'Department of Physical Therapy, College of Health Science, Student of Dankook University; '2Department of Physical Therapy, Dankook University, \\ Cheonan, Korea
}

Purpose: This study evaluated the usefulness of International Classification of Functioning, disability and health core set for children and youths with cerebral palsy (ICF-core set for CP) by comparing the Gross Motor Function Measure-88 (GMFM-88), which is the most widely used outcome measure in children with cerebral palsy (ICF-core set for CP).

Method: One subject (Female, 14) was evaluated by GMFM-88 and ICF-core set for CP. In addition, the concept of GMFM-88 was compared with the concept of the ICF-core set for CP in compliance with ICF linking rules. The numerical values both of the GMFM-88 categories and the ICF-core set for CP's items were compared.

Results: The ICF b760 was linked to the GMFM-88 59 61. d415 was linked to 57 58 of GMFM-88. d450 was linked to GMFM-88's 65 72. d455 was linked to GMFM-88 at 77and 80 83.

Conclusion: The association of the ICF-core set for CP and GMFM-88 was confirmed. In addition, the number of categories of ICF-core set for $\mathrm{CP}$ was smaller than the number of items of GMFM-88. In conclusion, the ICF-core set for CP is an evaluation tool that can identify the various features of children. In addition, GMFM-88 was linked to the ICF core set for CP according to the ICF linking rules.

Keywords: ICF-core set, Cerebral Palsy, ICF

\section{서 론}

뇌성마비(cerebral palsy)는 생후 2년 이하의 미성숙한 뇌에서 발생한 비진행성 손상으로 운동 및 자세 장애를 초래하는 뇌병변 장애이다. ${ }^{1}$ 뇌성마비는 활동의 제한을 초래하며 감각, 인지, 의사 소통, 지각, 시 각 등 다양한 기능에 이상을 동반한다. 따라서 뇌성마비를 가진 아 동의 기능적 수준을 판단하고 삶의 질을 향상시키기 위해서는 다양 한 분야의 평가가 필요하다.

그러나 국내는 물론이고 국외에서도 뇌성마비 평가도구의 표준화 는 이루어지지 않았다. 소아물리치료사 130명을 대상으로 진행한 설 문에 따르면 국내 뇌성마비 아동 평가에 사용되는 도구들은 주관적 서술이 47.1\%로 제일 높았으며, 대동작기능평가(gross motor function measure, GMFM)가 $21.3 \%$, 기관 내 자체 제작 도구가 $18.8 \%$ 순으로 사 용되었다. 국외 조사에서 32 명의 의료전문가들은 뇌성마비 아동의 평가에 103 개의 평가도구를 사용하였으며, 33 명의 의료 전문가들을 대상으로 해당 평가도구들의 선호도를 조사한 결과 72 개의 평가 도

Received Mar 19, 2019 Revised Apr 18, 2019

Accepted Apr 19, 2019

Corresponding author Hyoung won Lim

E-mail movt12@hanmail.net
구는 기준점을 넘지 못하였으며, 제일 선호하는 평가도구는 GMFM 이라고 보고 하였다. 또 다른 체계적 고찰에 따르면 14 개의 실험에 사 용된 구조적, 기능적 측정 도구는 16 개였으며 제일 많이 사용된 도구 는 GMFM이었다. 많은 치료사들이 환자의 특성을 확인하고 중재의 방향을 결정하기 위하여 평가도구 사용을 권장하지만 표준화된 평 가도구가 부족한 실정이다.

이와 같은 이유로 세계보건기구(world health organization, WHO)에 서는 건강과 건강관련 상태를 통일되고 표준화된 언어와 체계로 표 현할 수 있도록 국제 기능, 장애 그리고 건강분류(international classification of functioning, disability and health, ICF)를 개발하였다.5.6 ICF는 인류의 건강 및 건강관련 영역을 개인의 건강상태와 연관하여 신체 적, 개인적, 사회적 측면에서 폭 넓게 설명하고 있다.

ICF 분류는 신체 기능과 신체구조, 활동과 참여, 환경요인, 개인요 인 4 개의 요소로 구성된다. ICF의 세가지 구성요소(신체 기능과 구조, 활동과 참여, 환경요인은)는 장(chapter)으로 처음 분류되며 하위에 항목들(cate gories)이 두 번째, 세 번째, 네 번째 수준으로 배열된다.6.7

Copylight (C)2019 The Korean Society of Physical Therapy

This is an Open Access article distribute under the terms of the Creative Commons Attribution Non-commercial License (Http:// creativecommons.org/license/by-nc/4.o.) which permits unrestricted non-commercial use, distribution, and reproduction in any medium, provided the original work is properly cited. 
개인 요인은 아직 ICF에 분류되지 않았다.6,7 각 항목 들은 알파벳과 숫자로 코드화 된다. 머리글자 b, s, d, e로 어느 영역인지 표시하고 첫 번째 수준은 하나의 숫자 코드로 두 번째 수준은 3 개의 숫자 코드로 세 번째는 4 개, 네 번째는 5 개로 나타낸다. 이후 WHO는 18 세 이하의 아동 및 청소년을 위해 기존 ICF에 아동 청소년과 관련된 항목을 추 가하고 내용을 변경하여 아동 청소년을 위한 ICF인 ICF-CY (International classify- cation of functioning, disability and health: children \& youth version)를 개발하였다. ${ }^{8} \mathrm{ICF}$ 는 개인의 특성을 설명하기 좋은 평 가도구이지만, 기존의 ICF는 1,424 개의 항목이며, ICF-CY는 1,685 개 의 항목으로 이루어져 있기 때문에 임상실무와 연구에서 사용하기 에는 제한이 따른다. 이런 단점을 보완하고 일상 업무에서 사용하기 위하여 최근 $\mathrm{WHO}$ 는 다양한 건강 상태(health condition)와 질병 그 룹(condition group), 그리고 건강 관리 상황(health care context)에 따 라 기능을 체계적이고 포괄적으로 기술할 수 있는 core set 들이 개발 되고 있다. ${ }^{79} \mathrm{ICF}$-core set는 실용적으로 사용할 수 있도록 가장 적은 수로 구성된 ICF항목들의 목록이다. ${ }^{10}$ 각 항목들은 기능과 장애를 평 가하는 사용자를 위한 도구를 제공 하고자 과학적 절차를 통해 ICF 의 전체 항목에서 선별된다. ${ }^{710}$

18 세 이하의 뇌성마비 아동 및 청소년을 대상으로 사용할 수 있는 ICF-core set (ICF- core sets for children and youth with cerebral palsy, ICFcore set for CP)이 2014년에 발표되었다. ${ }^{9}$ ICF-core set for CP는 아동 및 청소년을 대상으로 개발된 최초의 core set이며 아동과 청소년의 성장, 발달을 고려하여 만들어졌다. 또한, 뇌성마비 아동과 청소년의 주요 특성과 관련성을 파악할 수 있으면서도 간결하고 정확한 항목으로 이 루어져 있다. ICF-core set for $\mathrm{CP}$ 는 여러 전문분야의 개입이 필요한 뇌 성마비 아동에 대해 아동의 기능 상태를 기술할 때 공통 언어로 사용 될 수 있고, ${ }^{7,911}$ 뇌성마비 집단의 표준화된 기능 정보 체계로 사용할 수 있어 여러 연구들을 용이하게 비교할 수 있을 것이라 기대된다. ${ }^{9}$ 뇌성 마비 아동에게 표준화된 ICF-core set for CP을 사용한다면, 뇌성마비 아동의 사례관리가 원활하게 이루어질 수 있을 것이다. 또한, 치료에 서의 다학문적인 접근을 가능하게 할 것이다. 이는 뇌성마비에 사용 되는 중재 향상을 위한 연구와, 뇌성마비 대상자와 가족에 대한 지원 에 바탕이 되는 복지법 및 정책의 근거가 될 수 있을 것이다.

국내외 뇌성마비 아동을 대상으로 삶의 질 평가나 중재 전후 변화 확인, 운동기능 평가에 ICF-CY나 체크리스트를 사용한 경우는 있지 만 ICF-core set을 활용한 사례는 매우 제한적이다. ${ }^{11,12}$ 이는 뇌성마비 아동을 위한 ICF-core set가 비교적 최근에 만들어졌기 때문으로 생 각된다. 따라서 본 연구에서는 학령기 뇌성마비 아동을 대상으로 GMFM과 ICF-core set을 측정하여, 가장 많이 활용되는 평가 도구인 GMFM과 비교하여 그 유용성을 알아보고자 한다.

\section{연구 방법}

\section{1. 연구 대상}

본 연구의 대상자는 제태기간 30 주에 제왕 절개로 분만하여 $1.44 \mathrm{~kg}$ 으로 태어났으며, 운동 영역인 뇌백질 영역에 경도의 뇌백질연화증 (periventricular leukomalacia)을 진단받은 만 14세 여아이다. 하지의 운 동 기능이 침범된 경직형 양하지 마비(spastic diplegia)로 하지의 근력 이 약하고 약한 경직이 있다. 상지의 운동 기능과 인지 기능은 침범되 지 않았다. 만 6세에 사타구니, 무릎 뒤, 종아리에 근육연장수술을 받 았으며, 주 2 회의 작업치료, 주 3 회 물리치료를 각각 한 시간 씩 총 5 시 간의 중재를 받고 있다. 엄마, 아빠, 언니와 함께 살고 있으며, 엘리베이 터를 이용 가능한 일반 중학교에 재학 중이다. 보행이 가능하며 전신 운동분류체계(gross motor function classification system, GMFCS)상 수준 II에 해당되었다. 대상자는 더 강한 힘과 안정성을 위하여 발목 보조기(ankle foot orthosis)를 사용한다.

\section{2. 평가도구}

\section{1) ICF-core set for $\mathrm{CP}$}

뇌성마비 아동을 위한 ICF-core set에는 크게 3가지 종류가 있다. 하나 는 종합적인 평가를 위한 상세판(comprehensive ICF-core set for CP)과 일반적인 영역만으로 축약한 일반 요약판(common brief ICF-core set for $\mathrm{CP}$ ), 마지막으로 연령별 특성을 추가한 연령 특이적 요약판(brief ICF-core set for $\mathrm{CP}$ )이다.

본 연구에서는 14-18세 아동을 위한 요약판(brief ICF-core set for CP14-18 years of age)에 집단과 개인의 기능과 건강 상태를 평가할 수 있는 공통판(ICF generic set)을 추가하여 사용하였다. ${ }^{13}$ 단 7 개의 ICF 항목 b130 (에너지와 구동기능), b152 (감정기능), b280 (통증감각), d230 (일상생활 수행하기), d450 (걷기), d455 (이동하기), d850 (유급 고용)로 구성된 공통판 만으로도 환자의 기능 수준을 한 눈에 이해하고 비교 할 수 있기에 ICF-core set를 사용할 때 함께 사용하도록 권장한다.

모든 항목은 손상의 정도를 숫자로 결과값(qualifiers)을 부여하여 사용하였다. 신체 기능과 신체 구조 영역의 점수는 $0=$ 손상 없음, $1=$ 경도 손상, $2=$ 중도 손상, $3=$ 고도 손상, $4=$ 완전 손상, $8=$ 분류되어 있 지 않음, $9=$ 적용 불가로 평가한다. 활동과 참여 영역은 $0=$ 어려움 없 음, $1=$ 경도 어려움, $2=$ 중도 어려움, $3=$ 고도 어려움, $4=$ 완전 어려움, $8=$ 분류되어 있지 않음, $9=$ 적용 불가 평가한다. 환경요인은 $0=$ 방해/ 촉진요인 없음, 1 = 경도 방해요인, 2 = 중도 방해요인, $3=$ 고도 방해요 인, $4=$ 완전 방해 요인, $+1=$ 경도 촉진 요인, $+2=$ 중도 촉진 요인, $+3=$ 고도 촉진 요인, $+4=$ 완전 촉진 요인으로 평가한다. 기록지와 기능 파 일은 ICF 홈페이지에서 제공하는 문서 양식을 사용하였다. ${ }^{14}$ 평가는 인터뷰 형식으로 진행되었으며 대상자가 스스로 느끼기에 불편함이 
어느 정도인지 응답하였다.

\section{2) GMFM-88}

GMFM-88은 뇌성마비와 아동의 대동작 기능을 평가하기 위해 국내 외에서 가장 보편적으로 사용되 고 있는 도구이다. 88 개 항목의 GMFM 은 뇌성마비아동의 움직임을 관찰한 후, 4 점 척도 $(0=$ 움직임을 시작 하지 않음, $1=$ 움직임을 시작하지만, 기능적 활동의 $10 \%$ 이내 수행, $2=$ 기능적 활동의 $10 \%$ 에서 $100 \%$ 미만으로 수행, $3=$ 기능적 활동을 완 벽하게 수행, $\mathrm{NT}=$ 평가 못 함)로 점수를 매긴다. 평가 항목은 A. 누운 자세(lying and rolling), B. 앉은 자세(sitting), C. 네발 기기(crawling)와 무릎서기(kneeling) D. 서기(standing) E. 걷기(walking)와 뛰기(running) 및 깡총뛰기(jumping) 활동의 5개 영역으로 구성되어 있다. 각 영역의 점수는 퍼센트 값으로 제시되며, 총점은 각 영역의 퍼센트 값 의 합을 5 로 나누어 제시하였다.

뇌성마비 아동의 평가에서 GMFM- 88 은 신뢰도(r=0.77 or 0.88$)$ 가 높은 평가 도구이다. ${ }^{15}$ 본 연구에서는 대상자가 보행이 가능하고 GMFCS II단계였기에, GMFM-88 D와 E영역 만을 평가 하였다. ${ }^{16,17}$

\section{3. 연구절차}

1)평가

연구 시작 전 보호자와 아동에게 연구 목적을 설명하고 참여에 동의 를 받아 2018년 7월 19일에 평가를 진행하였다. ICF-core set평가는 국 제건강기능분류 온라인 학습 도구(ICF e-learning tool)를 통해 충분 히 숙지한 이후 실시하였다.18

\section{2) 자료분석}

2002년 Cieza 등 ${ }^{19}$ 은 ICF를 다른 평가도구들과 연관시키는 방법(ICF linking rules)에 대한 규칙을 발표하였고 2007년 에 추가(upgrade) 규 칙을 발표하였다. ${ }^{20} \mathrm{ICF}$ 연관 규칙은 다른 평가도구들과 ICF 항목들의 연관성을 확인하기 위해 여러 번 사용된 바 있다.21-26 본 연구에서는 이 ICF연관 규칙을 준수하며 GMFM항목에서 핵심개념을 추출하였 고 추출한 개념과 가장 동일하다고 생각하는 ICF-core set for CP의 범 주들을 연관시켰다. GMFM-88의 53과 56항목처럼 제한시간을 둔 것 은 핵심개념에 포함시키지 않았다. 이후 GMFM항목의 평가 값과 그 항목과 연관된 ICF항목의 평가 값을 비교하여 수치가 비슷한 항목 만을 선별하였다. 두 과정 모두 연구자들이 각각 진행하였으며, 결과 를 서로 비교하고 논의하여 합의하였다.

\section{결 과}

\section{ICF-core set for CP 평가 결과}

ICF-core set for CP의 평가 결과는 다음과 같다. 신체기능에서 b210 (시 각기능), b735 (근 긴장도 기능), b760 (수의적 움직임 조절기능) 항목에 경도 손상이 있는 것으로 평가되었고 b280 (통증 감각)항목에 중도 손상이 있는 것으로 평가되었다. 유일한 신체 구조 영역인 $\mathrm{s} 110$ (뇌 구 조)항목은 중도 손상이 있는 것으로 평가되었다. 활동과 참여 영역에 서 $\mathrm{d} 230$ (일상 생활수행하기)은 경도 어려움이, $\mathrm{d} 415$ (자세 유지하기) 은 중도 어려움, $\mathrm{d} 455$ (이동하기)은 고도 손상이 있는 것으로 평가되 었다. $\mathrm{d} 845$ (구직하기, 근속하기 및 퇴직하기)와 $\mathrm{d} 850$ (유급 고용)은 측 정할 수 없는 손상이 있는 것으로 평가되었다. 환경 요인에서 e460 (사 회적인 태도)은 중도 방해요인, $\mathrm{e} 150$ (공용 건물의 설계, 시공과 건축 물 및 기술), e580 (보건서비스, 시스템 및 정 책)은 고도 방해 요인, e540 (교육 서비스, 시스템 및 정책), e585 (교육과 훈련 서비스, 시스템 및 정책)는 완전 방해 요인으로 평가되었다(Table 1).

\section{GMFM-88 평가 결과(D 와 E 영역)}

GMFM-88 의 평가 결과는 다음과 같다. D 영역은 31 점(79\%) 이였으며, $\mathrm{E}$ 영역은 점수 63 점(88\%)을 나타냈다(Table 2).

\section{ICF-core set for CP와 GMFM-88 비교}

ICF 연관규칙에 따라 비교한 결과 총 36 개의 GMFM 항목이 ICF항목 (category) 4 개와 연관성이 확인되었다. b760수의적 움직임 조절은 GMF M-88 의 59-64, 78, 79번과 연관되었고, d415자세유지는 GMFM88의 53-58번과, d450보행은 GMFM- 88 의 65 번-74번과, d455이동 하기 는 GMFM-88의 75-77번, 80-88번과 연관되었다. 연관되지 않은 항목 들은 ICF core set에 적절한 개념이 없는 항목들이었다.

연관된 항목들의 결과값을 비교하여 다시 한번 연계시킨 결과 b760수의적인 움직임 조절 기능과 GMFM-88의 59-62번이 연관 있었 고, d415신체자세 유지하기는 GMFM-88의 57-58번, d450보행은 GMFM-88의 65-72번과 d455주변 이동하기는 GMFM-88의 77번, 80-83번과 연관 있는 것으로 나타났다(Table 3 ).

\section{고 찰}

본 연구는 뇌성마비 아동을 평가하는 도구로 ICF-core set for CP사용 을 제안하기 위해, 현재 뇌성마비 아동의 평가에 가장 일반적으로 사 용되는 평가 도구인 GMFM-88과 비교하였다. Cieza ${ }^{19,20}$ 등이 발표한 ICF연관 규칙에 따라 GMFM-88 의 D, E영역의 항목(items)을 각 항목 별로 움직임 조절(movement control), 자세 유지(maintaining position), 보행(walking), 이동하기(moving)등의 핵심 개념 (concept)을 추출하고 가장 적절한 ICF-core set for CP의 항목(category)과 연관시켰다. 그 결 과 총 36 개의 GMFM항목이 ICF항목 4개와 연관성이 확인되었다. 본 
Table 1. ICF-core set for CP

\begin{tabular}{|c|c|c|c|c|c|}
\hline \multirow{2}{*}{ Body Functions } & \multicolumn{5}{|c|}{ impairment } \\
\hline & 0 & 1 & 2 & 3 & 4 \\
\hline \multicolumn{6}{|l|}{ b117 intellectual functions } \\
\hline \multicolumn{6}{|l|}{ b130 energy and drive functions $(\mathrm{G})$} \\
\hline \multicolumn{6}{|l|}{ b1301 motivation } \\
\hline \multicolumn{6}{|l|}{ b134 sleep functions } \\
\hline \multicolumn{6}{|l|}{ b152 emotional functions (G) } \\
\hline \multicolumn{6}{|l|}{ b164 higher-level cognitive functions } \\
\hline \multicolumn{6}{|l|}{ b167 mental functions of language } \\
\hline \multicolumn{6}{|l|}{ b210 seeing functions } \\
\hline \multicolumn{6}{|l|}{ b280 sensation of pain $(G)$} \\
\hline \multicolumn{6}{|l|}{ b710 mobility of joint functions } \\
\hline \multicolumn{6}{|l|}{ b735 muscle tone functions } \\
\hline b760 control of voluntary movement function & & & & & \\
\hline
\end{tabular}

\begin{tabular}{lllll}
\hline \multirow{2}{*}{ Body Structures } & \multicolumn{3}{c}{ impairment } \\
\cline { 2 - 4 } & 0 & 1 & 3 & 4 \\
\hline s110 structure of brain & \multicolumn{3}{c}{ limitation } \\
\hline Activities and Participation & 0 & 1 & 3 & 4 \\
\hline
\end{tabular}

d175 solving problems

d230 carrying out daily routine(G)

d250 managing one's own behavior

d415 maintaining a body position

d440 fine hand use

d450 walking (G)

d455 moving around (G)

d460 moving around in different locations

d530 toileting

d550 eating

d570 looking after one's health

d710 basic interpersonal interactions

d720 complex interpersonal interactions

d760 family relationships

d820 school education

d845 acquiring, keeping and terminating a job

d850 remunerative employment (G)

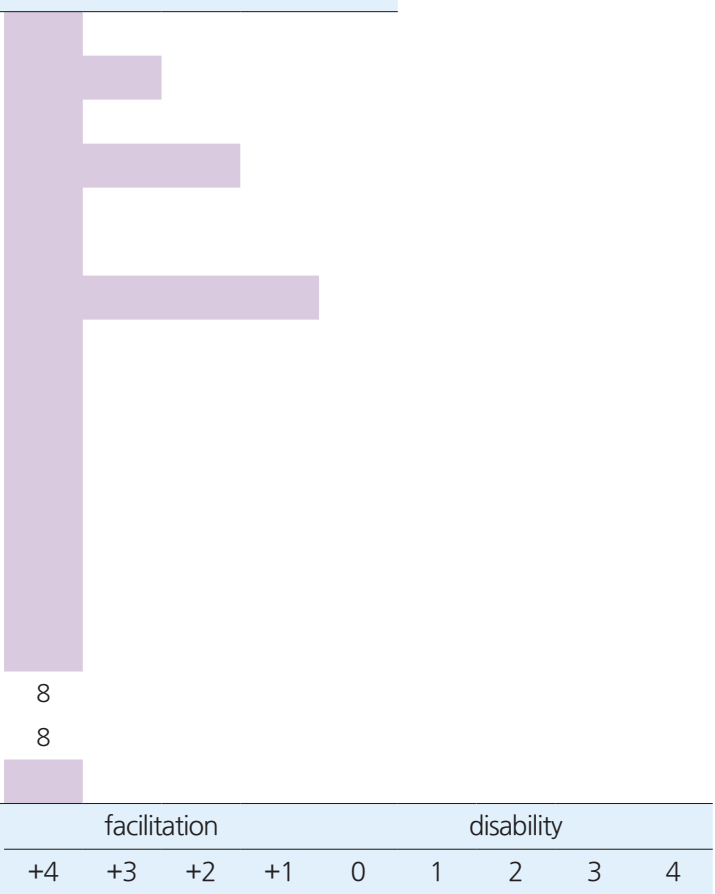

d920 recreation and leisure

Environmental Factors

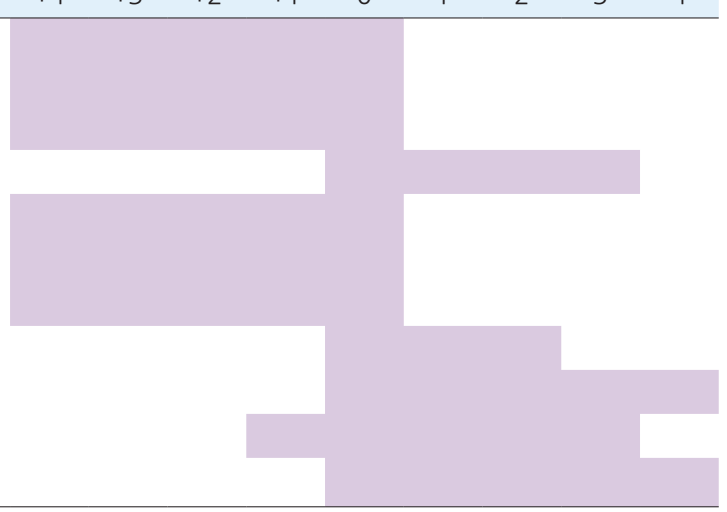

e115 products and technology for personal use in daily living

e120 products and technology for personal indoor and outdoor mobility and transportation

e125 products and technology for communication

e150 design, construction and building products and technology of buildings for public use

e310 immediate family

e320 friends

e420 individual attitudes of Friends

e460 social attitudes

e540 transportation services, systems and policies

e580 health services, systems and policies

e585 education and training services, systems and policies 
Table 2. GMFM-88

\begin{tabular}{|c|c|c|c|c|c|}
\hline \multirow{2}{*}{ D. Standing } & \multicolumn{5}{|c|}{ Score } \\
\hline & 0 & 1 & 2 & 3 & NT \\
\hline \multicolumn{6}{|c|}{ 52. on the floor: pulls to std at large bench } \\
\hline \multicolumn{6}{|l|}{ 53. STD: maintains, arms, free 3 seconds } \\
\hline \multicolumn{6}{|c|}{ 54. STD: holding on to large bench with on hand, lift R foot, 3 seconds } \\
\hline \multicolumn{6}{|c|}{ 55. STD: holding on to large bench with on hand, lift L foot, 3 seconds } \\
\hline \multicolumn{6}{|c|}{ 56. STD: maintains, arms, free 20 seconds } \\
\hline \multicolumn{6}{|l|}{ 57. STD: lift L foot. arms free, 10 seconds } \\
\hline \multicolumn{6}{|c|}{ 58. STD: lift R foot. arms free, 10 seconds } \\
\hline \multicolumn{6}{|c|}{ 59. sit on small bench: attains STD without using arms } \\
\hline \multicolumn{6}{|c|}{ 60. high KN: attains STD though half KN on R knee, without using arms } \\
\hline \multicolumn{6}{|c|}{ 61. high $\mathrm{KN}$ : attains STD though half $\mathrm{KN}$ on $\mathrm{L}$ knee, without using arms } \\
\hline \multicolumn{6}{|c|}{ 62. STD: lowers to sit on floor woth control, arms free } \\
\hline \multicolumn{6}{|l|}{ 63. STD: attains squat. Arms free } \\
\hline \multicolumn{6}{|c|}{ 64. STD: pick up object from floor, arms free, returns to stand } \\
\hline \multirow{2}{*}{ E. Walking, running and jumping } & \multicolumn{5}{|c|}{ Score } \\
\hline & 0 & 1 & 2 & 3 & NT \\
\hline
\end{tabular}

65. STD: 2 hand on large bench: cruises 5 stepto $R$

66. STD: 2 hand on large bench: cruises 5 stepto $L$

67. STD: 2 hand held: walks forword 10 steps

68. STD: 1 hand held: walks forword 10 steps

69. STD: walks forword 10 steps

70. STD: walks forword 10 steps, stops, turns $180^{\circ}$, returns

71. STD: walks backword 10 steps

72. STD: walks forword 10 steps, carrying a large object with 2 hand

73. STD: walks forword 1010 consecutive steps between parallel line $20 \mathrm{~cm}$ apapart

74. STD: walks forword 1010 consecutive steps on a straight line $2 \mathrm{~cm}$ wide

75. STD: steps over stick at knee level, $R$ foot leading

76. STD: steps over stick at knee level, L foot leading

77. STD: run $4.5 \mathrm{~cm}$ sports and returns

78. STD: kick ball with $R$ foot

79. STD: kick ball with $L$ foot

80. STD: jumps $30 \mathrm{~cm}$ high, both high, both feet simultaneously

81. STD: jumps forward $30 \mathrm{~cm}$ both feet simulataneously

82. STD on R fOot: HOPS ON R FOOT 10 TIMES WITHIN A $60 \mathrm{~cm}$ CIRCLE

83. STD on L FOOT: HOPS ON L FOOT 10 TIMES WITHIN A $60 \mathrm{~cm}$ CIRCLE

84. STD holding 1 rail: walks up 4 steps holding 1 rail alternating feet

85. STD holding 1 rail: walks down 4 steps holding 1 rail alternating feet

86. STD: walks up 4 steps. Alternating feet

87. STD: walks down 4 steps alternating feet

88 . STD on $15 \mathrm{~cm}$ step: jumps off both feet simulataneously

NT: not test, STD: standing, R: right, L: left, KN: kneeling.

Table 3. Comparing ICF-core set for CP and GMFM-88

\begin{tabular}{lll}
\hline ICF-core set for CP & \multicolumn{1}{c}{ Concept } & GMFM-88 \\
\hline b760 control of voluntary movement function & Movement control & $59-62$ \\
d415 maintaining a body position & Maintaining position & 57,58 \\
d450 walking (G) & Walking & $65-72$ \\
d455 moving around (G) & Moving & $77,80-83$ \\
\hline
\end{tabular}


연구는 이에 그치지 않고 연관된 항목들을 결과값으로 한번 더 비교 하여 연관성을 찾았다. 최종적으로 GMFM-88의 19 개의 항목을 ICFcore set for $\mathrm{CP}$ 의 4 개의 항목과 연관시켰다.

Schiariti 등 25 이 ICF 연관 규칙대로 뇌성마비 아동 및 청소년을 위 한 ICF-core set의 항목과 기존의 임상 평가도구들을 연관시킨 연구에 서, GMFM-88과 연관성 있는 ICF의 항목은 b760, d415, d450으로 밝 혀졌으며, 이는 본 연구 결과와 일치한다. 그러나, $\mathrm{d} 455$ 는 선행 연구에 서 사용한 ICF-core set에는 없었으며 공통판에 포함되는 항목이었기 때문에 본 연구에서만 연관성이 있는 것으로 나타났다. 이전 연구에 서 연관규칙에 따라 GMFM-88의 항목을 ICF-CY 의 전 항목과 연관 시킨 결과, 88 중 81 개의 항목이 d4이동성 영역의 항목과 연관이 있는 것으로 밝혀졌다. ${ }^{26}$ 이 선행연구에서는 $\mathrm{d} 450$ (보행)과 하위의 항목은 GMFM-88의 항목 10 개와 연관되었고, 이는 본 연구에서 연관규칙으 로 $\mathrm{d} 450$ 과 연관시킨 결과와 동일 하다. 반면, 선행연구에서 $\mathrm{d} 415$ (자세 유지)와 하위 항목은 8개 GMFM항목과 연관되었으며, $\mathrm{d} 455$ (이동하 기)와 하위항목은 21 개의 GMFM항목과 연관되었다. 본 연구에서 ICF 연관규칙 대로 핵심개념을 추출 하였을 때, $\mathrm{d} 415$ 와 6 개의 항목이 연관되었으며, $\mathrm{d} 455$ 는 12 개의 항목과 연관되었다. 선행연구는 본 연 구의 결과보다 많은 수의 GMFM항목과의 연관성을 나타냈다. 이러 한 이유로는 사용한 GMFM의 총 항목 수가 다르기 때문이라고 생각 된다. 즉, GMFM의 D 와 E영역 만을 사용한 본 연구에 비해 A, B, C영 역까지 포함시킨 이전 연구의 GMFM항목 수가 더 많았기에 나타난 결과로 설명할 수 있다.

본 연구의 대상자는 영상의학적 검사상 하지의 운동영역을 담당 하는 백질 부위가 손상된 것으로 나타났다. 이는 수의적 운동을 가 능하게 함과 동시에 선택적 운동조절(selective motor control) 또한 담 당하는 하행 운동 신경로인 피질척수로(corticospinal tract, CST)가 손 상되었음을 보여주는 것이다. ${ }^{27}$ 이러한 결과는 대상자에게 근력 약화 와 경직(spasticity), 근 길이의 단축 발생을 유추할 수 있으며, 실제로 대상자에게 동일한 문제점이 있다고 확인되었다. 따라서 본 연구의 대상자는 b760(수의적 움직임 조절) 능력이 손상된 것으로 평가 할 수 있다. 선택적 운동조절 능력은 GMFM 과도 높은 상관관계가 있 다. 28,29 따라서 b760(수의적 움직임 조절)과 GMFM은 연관되었다고 할 수 있다. d415(자세유지)는 책상에 앉아 수업을 듣는 등 한 자세를 유지해야 할 때 겪는 어려움의 정도를 평가하는 항목이다. 본 대상자 는 2점으로 평가되었다. 연관 규칙을 통해 $\mathrm{d} 415$ (자세 유지하기)와 연 관성이 확인된 GMFM 의 항목 중 평가 결과가 유사한 2 개의 항목은 각 1점으로 평가되었다. 이는 균형 부족 때문이라 생각된다. 선행 연 구에서 균형과 GMFCS간의 높은 상관관계가 보고되었으며, ${ }^{30,31}$ GMFCS와 GMFM역시 높은 상관관계가 있다고 보고 되었다. ${ }^{32}$ 따라 서 균형과 GMFM도 상관관계가 있다고 생각된다. 그러므로 $\mathrm{d} 415$ 자
세유지는 정적 균형의 일부로서 GMFM과 연관이 있다고 생각된다. 탈락된 53-56번은 한 손으로 지지하거나 두발로 서기 등 상대적으로 난이도가 낮은 항목이었다. ${ }^{33}$ 따라서 보조기의 도움 없이 독립 보행 을 할 수 있는 대상자가 충분히 할 수 있었던 것으로 생각된다.

ICF항목 $\mathrm{d} 450$ 은 보행의 형태나 질과는 관계없이 실생활에서의 보 행 여부를 평가하는 항목이다. 반면, GMFM은 보행의 형태나 질과 연 관이 있다고 보고 되었다. ${ }^{34,35}$ 본 연구에서 ICF연관 규칙으로 $\mathrm{d} 450$ (보 행)과 연관된 GMFM-88항목 10 개 중 2개는 점수가 낮아 제외되었다. 제외된 항목은 문제없이 수행한 다른 항목보다 수행하기 더 어려운 항목이라고 보고 되었다. ${ }^{33}$ 따라서 GMFCS II단계로 독립 보행을 할 수 있는 본 연구의 대상자가 보행의 어려움의 정도를 평가하는 $\mathrm{d} 450$ 은 0 점으로 평가되면서 GMFM의 난이도가 높은 보행 항목은 3점보 다 낮은 점수를 받은 것으로 생각된다.16

형태와 질을 평가하지 않은 ICF항목은 $\mathrm{d} 450$ 만이 아니다. $\mathrm{d} 455$ 는 자전거 타기, 수영하기, 계단 오르기 등 보행을 제외한 이동에서의 어 려움을 평가한다. 대상자는 계단을 이용 할 수 있지만 (4층까지 계단 으로 이동 가능) 사람이 많아 복잡하면 엘리베이터를 이용하고, 자전 거 타기, 수영하기 등은 아예 수행하지 못하고 학교 체육시간에도 참 가하지 않는다고 하였기에 3점으로 평가되었다. GMFM 의 75,76 항목 은 장애물 넘기로 오르기(climbing)에 해당된다. 따라서 $\mathrm{d} 455$ 와 연관 성이 있다고 할 수 있다. GMFCS II단계의 아동은 4 세 이후라면 잡거 나 보조를 받아 계단을 오르고 내릴 수 있다. ${ }^{16}$ 본 연구의 대상자는 난 간을 잡은 상태는 물론이고 난간과 보조 없이도 4계단을 오르고 내 릴 수 있었다. 이는 본 연구의 대상자가 이미 보조도구 없이 걸어 다닐 수 있는 비교적 기능이 좋은 아동이기 때문에 가능한 것이라고 생각 된다.

선행연구에서 뇌성마비 아동의 운동 기능은 아동의 다양한 특성 의 영향을 받는다고 하였다. ${ }^{36,37}$ 따라서 뇌성마비 아동을 평가할 때, 운동 기능뿐 만 아니라 다른 영역도 포함하여 종합적이고 다학문적 인 접근을 통해 평가해야 한다. ICF-core set for CP는 42개 항목 안 에 아동의 성격과 특성, 가족과의 관계, 사회와의 상호관계 등 다양한 영 역을 포함하고 있다. 따라서 ICF-core set for CP를 뇌성마비 아동의 재 활에서 사용한다면 적은 수의 항목으로 아동의 운동 기능뿐만 아니 라 아동의 다양한 특성을 평가하고 특성 간의 상호작용을 고려하여 중재를 계획할 수 있도록 도울 수 있을 것이다.

마지막으로 현재 국내 장애 인 활동 지원제도는 소득수준, 연령, 장 애 등록 여부, 수정된 바델 지수 (modified bathel index, MBI)에서 ICF 를 반영하는 추세로 바뀌고 있다. 그 외에도 활동인정 조사표는 일상 생활동작(activities of daily living, $\mathrm{ADL}$ )과 수단적 일상생활 동작(instrumental activities of daily living, IADL)을 기준으로 사용하고 ICF를 기본 바탕으로 만들어져 활용하고 있다. 2019년 7월 이후 장애등급제 
도와 대체되어 복지제도의 기준으로 시행될 예정인 장애인 종합지원 조사표 역시 ICF의 전 구성요소(b, s, d, e)를 바탕으로 개발 되었으며, $\mathrm{ICF}$ 에서 사용되는 척도와 교차 가능하다. 이처럼 최근에 개발되는 장애인 서비스 기준은 ICF를 적극 반영하고 있다. 그러므로 임상에 서 뇌성 마비 아동을 평가할 때, ICF- core set for CP를 활용한다면 정 책과 더불어 지원조사표 활용에도 용이할 것이다.

본 연구에서는 ICF-core set for CP가 GMFM-88과 연관이 있는 것 으로 나타났다. 현재 국내외에서 ICF는 ICD (international classification disease)와 병행해서 사용할 수 있으며, 개인과 집단의 건강상태 를 측정하고 표시하는 도구로서 활용을 권장하고 있다. 그럼에도 불 구하고 임상에서 ICF를 사용하 기에는 항목이 많아 어려움이 있다. 그러나, ICF-core set for CP는 항목 수가 적으면서 운동기능과 함께 아 동의 다양한 특성을 평가할 수 있으므로 사용을 권장하는 바이다.

본 연구의 제한점은 다음과 같다. 첫째, 대상자가 한 명이기 때문에 본 연구가 뇌성 마비의 일반적인 특성을 대표한다고 할 수 없는 점이 다. 둘째, 현재 뇌성마비 아동 및 청소년을 위한 ICF-core set는 나중에 개발되어 공식적으로 번안되지 않아 사용에 제한을 갖는 점이다. 마 지막으로 ICF는 GMFM-88과 달리 정량화 되지 않고 항목화 되어 점 수를 연산할 수 없기에 절대 기준으로 사용하기에는 부적합하다는 점이다. 향후 연구에서는 다양한 연령대와 더 많은 아동 및 청소년을 대상으로 ICF-core set을 적용해야 할 것이다.

\section{REFERENCES}

1. M Bax, M Goldstein, P Rosenbaum et al. Proposed definition and classification of cerebral palsy, Dev Med Child Neurol. 2005;47(8):571-6.

2. HJ Park, CH Yi, SH Gho et al. Physical therapist's understanding and the usage of assessment and cerebral palsy. J Kor Phys Ther. 2000;7(1):1-21.

3. J N Vargus-Adams, L Martin. Measuring what matters in cerebral palsy: a breadth of important domains and outcome measures. Arch Phys Med Rehabil. 2009;90(12):2089-95.

4. R Kunz, I Autti-Ramo, H Anttila et al. A systematic review finds that methodological quality is better than its reputation but can be improved in physiotherapy trials in childhood cerebral palsy. J Clin Epidemiol. 2006;59(12):1239-48.

5. World Health Organization. International classification of functioning, disability and health. geneva: world health organization. 2001.

6. World Health Organization. International classification of functioning, disability and health. 2nd ed. Ministry of health \& welfare. 2016.

7. J Bickenbach, A Cieza, A Rauch et al. ICF-core sets: manual for clinical practice. Boston, Hogrefe Publish Cop. 2012.

8. World Health Organization, International classification of functioning, disability and health: children \& youth version: ICF-CY. World health organization. 2007.

9. V Schiariti, M Selb, A Cieza et al. International classification of functioning, disability and health core sets for children and youth with cerebral palsy: a consensus meeting. Dev Med Child Neurol. 2015;57(2):149-58.

10. G Stucki, A Cieza, T Ewert et al. Application of the international classification of functioning, disability and health (ICF) in clinical practice. Disabil Rehabil. 2002;24(5):281-2.

11. JH Kim, TH Kim. An ICF-Core sets for children and youth with cerebral palsy based approach from a physical therapist perspective: a single case study. J Kor Phys Ther. 2016;23(1):55-64

12. RP de Olivia, CACT Caldas, M Riberto. Application of the ICF-CY Brief Core Set for cerebral palsy on a school age child. Acta Fisiatr. 2016;19(3): 46-50.

13. A Cieza, C Oberhauser, J Bickenbach et al. Towards a minimal generic set of domains of functioning and health. BMC Public Health. 2014;14(3):218-26.

14. ICF, ICF-based documentation tool. https://www.icf-core-sets.org.

15. R Palisano, P Rosenbaum, S Walter et al. Development and reliability of a system to classify gross motor function in children with cerebral palsy. Dev Med Child Neurol. 1997;39(4):214-23.

16. Palisano RJ, Hanna SE, Rosenbaum PL et al. Validation of a model of gross motor function for children with cerebral palsy. Phys Ther. 2000; 80(10):974-85.

17. Song JY, Choi JD. The usability study for Gross Motor Function classification system as motor development prognosis in children with cerebral palsy. J Kor Phys Ther. 2008;20(1):49-56.

18. ICF, ICF e-learning tool, https://www.icf-elearing.com.

19. Cieza A, Brockow T, Ewert T eat al. Linking health-status measurements to the international classification on functioning, disability and health. J Rehabil Med. 2002;34:2005-210.

20. Cieza A, Gevh S, Chatterji S et al. ICF linking rules: an update based on lessons learned. J Rehabil Med. 2005;37(4):212-8.

21. Stucki G, Cieza A. The international classification of functioning, disability and health (ICF) core set for rheumatoid arthritis: a way to specify functioning. Ann Rheum Dis. 2004;63(2):40-5

22. JM Song, HJ Lee. Mapping items of functioning questionnaires into the international classification of functioning, disability and health: stroke. Kor Phys Ther. 2016;28(5);341-7.

23. HJ Lee, JM Song. Mapping items of functioning questionnaires into the international classification of functioning, disability and health: low back pain. Kor Phys Ther. 2016;28(5);321-7.

24. HJ Lee, JM Song. Linking of Items in two function-related questionnaires to the international classification of functioning, disability and health: shoulder pain. J Kor Phys Ther. 2018;30(6):239-45

25. Schiariti V, Tatla S, Sauve K et al. Toolbox of multiple-item measures aligning with the ICF-core sets for children and youth with cerebral palsy. Eur J Paediatr Neurol. 2017;21(2):252-63.

26. Engelen V, Ketelaar M, Gorter JW. Selecting the appropriate outcome in paediatric physical therapy: how individual treatment goals for children with cerebral palsy are reflected in GMFM-88 and PEDI. J Rehabil Med. 2007;29(3):225-31.

27. Fowler EG, Staudt LA, Greenberg MB. Lower-extremity selective voluntary motor control in patients with spastic cerebral palsy: increased distal motor impairment. Dev Med Child Neurol. 2010;52(3):264-9.

28. Lim HW. Correlation between the selective control assessment of lower extremity and pediatric balance scale scores in children with spastic cerebral palsy, J Phys Ther Sci. 2015;27(12):3645-49. 
29. Park EY, Kim WH. Structural equation modeling of motor impairment, gross motor function, and the functional outcome in children with cerebral palsy. Res Dev Disabil. 2013;34(5):1731-9.

30. Pavao SL, F.Barbosa KA, Tatiana de Oliveira Sato et al. Functional balance and gross motor function in children with cerebral palsy. Res Dev Disabil. 2014;35(10):2278-83.

31. Lim HW. Study of the usefulness of pediatric balance scale as a prediction indicator for gross motor function classification system in children with cerebral palsy. J Kor Phys Ther. 2016;2(1):22-6.

32. Effinger DJ, Tylkowski CM, Rayens MK et al. Gross motor function classification system and outcome tools for assessing ambulatory cerebral palsy: a multicenter study. Dev Med Child Neurol. 2004;46(5):311-9.

33. DJ Russell, LM Avery, PL Rosenbaumet al. Improved scaling of the gross motor function measure for children with cerebral palsy: evidence of reliability and validity. Phys Ther. 2000;80(9):873-85.

34. Lee JL, Cho SH, Kwon OY et al. The relevance between gross motor function measurement and the spatiotemporal parameters of gait in children with cerebral palsy. J Kor Phys Ther. 2001;8(1):20-34.

35. Damiano DL, Abel MF. Relation of gait analysis to gross motor function in cerebral palsy. Dev Med Child Neurol. 1996;38(5):389-96.

36. Bartlett DJ, Palisano RJ. A multivariate model of determinants of motor change for children with cerebral palsy. Phys Ther. 2000;80(6):598-614.

37. Kim JG, Lee JW. The effects of family support program on gross motor function, support system, and parenting stress in children with cerebral palsy. Journal of Rehabilitation Research. 2012;16(4):157-71. 